\title{
Effects of different anti-caries procedures on microhardness and micromorphology of irradiated permanent enamel
}

\author{
LiLing WU1,2, Kun GENG ${ }^{2}$ and QingPing GAO 2 \\ ${ }^{1}$ Department of Stomatology, Zhongshan Hospital Xiamen University, Xiamen 361004, Fujian Province, China \\ ${ }^{2}$ Center of Stomatology, Xiangya Hospital, Central South University, Changsha 410008, Hunan Province, China \\ Corresponding author, QingPing GAO; E-mail: gqp19722005@163.com
}

\begin{abstract}
To explore the effects of infiltration resin, casein phosphate polypeptide-amorphous calcium phosphate (CPP-ACP) and fluoride on microhardness and micromorphology of irradiated enamel. Sixty human permanent teeth were mesiodistally sectioned, yielding 120 enamel samples, which randomly divided into 8 groups: G1: blank control; G2: irradiation control; G3: irradiation+fluoride; G4: irradiation+CPP-ACP; G5: irradiation+CPP-ACP+fluoride; G6: irradiation+infiltration resin; G7: irradiation+infiltration resin+fluoride; G8: irradiation+infiltration resin+CPP-ACP. A progressive improvement was observed on the superficial morphology of enamel treated with different anti-caries procedures. The order of microhardness values after irradiation from the highest to the lowest was as follows: $\mathrm{G} 1>\mathrm{G} 8 \sim \mathrm{G} 7>\mathrm{G} 6>\mathrm{G} 5>\mathrm{G} 4 \sim \mathrm{G} 3>\mathrm{G} 2$. CPP-ACP, infiltration resin and fluoride can effectively restore the direct destruction of enamel caused by irradiation and promote the occurrence of remineralization. Infiltration resin and its combined effects with fluoride or CPP-ACP have the most potential anti-caries agent to resist radiation-caries.
\end{abstract}

Keywords: Radiotherapy, Permanent enamel, Infiltration resin, Casein phosphate polypeptide-amorphous calcium phosphate (CPP$\mathrm{ACP})$, Remineralization

\section{INTRODUCTION}

One of the most common complications in irradiated head and neck cancers (HNC) patients is a well-known devastating clinical finding of dental hard tissues known as radiation-related caries (RRC) ${ }^{1}$. RRC is a rapidly progressing and highly destructive type of dental caries, which is circumferential at the cervical part of the tooth, affecting smooth enamel surfaces that are normally resistant to decay, and then lead to the complete crown amputation of part of, or the whole, dentition ${ }^{2}$. There is evidence that the direct effects of radiotherapy on teeth are one of the important pathogenesis of RRC, which includes changes in microstructure and the mechanical properties of dental hard tissues ${ }^{3)}$. Fränzel and Gerlach $^{4)}$ found that enamel demineralization was shown after irradiation, accompanied with significantly changes of morphology and structure of enamel as well as the decrease of acid-resistance, which resulted in the increased sensitivity to caries. Because of the rapid progression of RRC and its difficulty of controlling, RRC is also known as 'rampant caries', whose treatment is mainly focus on prevention in clinical.

Kumar and Rastogi ${ }^{5)}$ found that oral hygiene instructions before and after radiotherapy in $\mathrm{HNC}$ patients can effectively reduce the incidence and severity of RRC. Fluorides, whose effects on caries has been well established in clinical trials and systematic reviews, can effectively prevent the damage of enamel caused by radiotherapy and be a feasible measure to prevent the occurrence of RRC in irradiated HNC patients ${ }^{6}$. For the last decade, a novel product casein phosphate polypeptide-amorphous calcium phosphate (CPP-ACP), which is a nanocomplex derived from milk protein and usually used for the management of incipient caries, has become commercially available ${ }^{7}$. CPP contains the cluster sequence of $\operatorname{Ser}(\mathrm{P})$-Ser(P)-Ser(P)-Glu-Glu from casein and is reported to have topical anti-caries effects due to its ability to stabilize calcium and phosphate in an amorphous state. CPP-ACP affects the demineralizationremineralization processes by maintaining an hypersaturated state on the tooth surface and a reservoir of calcium phosphate ${ }^{8}$. Its anti-cariogenic properties and remineralized effects have been demonstrated in animal laboratory ${ }^{9)}$, in vitro studies ${ }^{10)}$ and human in situ experiments ${ }^{7}$. However, the reported anti-caries efficacy of CPP-ACP in comparison with traditional fluorides was controversial ${ }^{7}$. In a randomized clinical trial $^{11)}$ of 24 patients with nasopharyngeal carcinoma (NPC), it was found that patients with CPP-ACP plus fluorides showed lower caries progressive behavior than those with fluorides alone. However, this synergistic remineralization effects of fluorides and CPP-ACP was not observed in other studies ${ }^{12,13)}$. Therefore, whether fluorides and CPP-ACP have combined effects on remineralization is still controversial.

Infiltration resin is a recent approach to limit enamel demineralization. This material was originally used in clinical to prevent the progression of noncavitated dental caries and later considered as an alternative treatment for fluorides and $\mathrm{CPP}-\mathrm{ACP}^{144}$. Resin infiltration technique can slow down or reverse the progress of non-cavitated enamel lesions and has preventive effects on rampant caries caused by Sjogren syndrome ${ }^{15)}$. In addition, infiltration resin has combined effects with CPP-ACP and fluorides in caries-prevention. Inhibition of caries progression in permanent teeth was significantly effective after treatment of resin 
infiltration and fluoride varnish than that of resin infiltration or fluoride alone ${ }^{16)}$. Chen ${ }^{17)}$ found that the combined treatment better improved the effects both in remineralization and inhibition of demineralization than the application of resin infiltration or CPP-ACP alone. To the best of our knowledge, whether this effect will be the same when infiltration resin applied alone or combined with CPP-ACP or fluoride to irradiated enamel is still unknown.

Therefore, the purpose of this study was to evaluate, by scanning electron microscopy (SEM) and micro indentation hardness test, the in vitro ability of different anti-caries procedures to prevent damage of dental hard tissues caused by radiotherapy. The null hypothesis tested was that different anti-cariogenic agents does not influence the micromorphology and microhardness of irradiated permanent enamel.

\section{MATERIALS AND METHODS}

A total of sixty healthy human permanent premolars and molars were collected from Center of Stomatology of Xiangya Hospital of Central South University after suitable approval from the Human Research Ethics Committee of the same institution (20171117) and performed as a double-blind study. The teeth were extracted for orthodontic reasons and none of them had any caries, restorations, surface defects, cracks, or enamel hypoplastic. The teeth were thoroughly washed, scraped, and ultrasonically scaled, to remove plaque and calculus, then stored in physiological saline solution at a temperature of $4^{\circ} \mathrm{C}(\mathrm{DW}-25 \mathrm{~W} 322$, Aucma, Qingdao, China) and used not more than 3 months postextraction ${ }^{18)}$.

\section{Sample preparation ${ }^{19)}$}

The teeth were cleaned and had their roots removed at the cementum-enamel junction (CEJ) using a highspeed diamond disc (MANI, Tochigi, Japan) under cooling-water. Crowns were sectioned longitudinally with a water-cooled diamond saw into two halves in the mesiodistal direction, yielding 120 enamel samples. The buccal or lingual surfaces were used as tested surfaces and ground with an increasing sequence of silicon carbide (SiC) paper $(1500,2000,2500,3000,5000$ and 7000 grit) for $60 \mathrm{~s}$ each under running water to produce flat enamel. Between each polishing stage, samples were cleaned using physiological saline solution in an ultrasonic cleaner (JP-010T, Jiemeng, Shenzhen, China) for $8 \mathrm{~min}$ to remove any debris. These 120 samples were randomly divided into eight groups $(n=15)$. All samples were embedded with medical red wax and exposed the tested surface, which was covered with two layers of acid-resistant nail polish, leaving a $3 \times 3 \mathrm{~mm}$ window exposed for microhardness test and SEM observation.

\section{Irradiation procedure}

The radiation dose was calculated based on a mean that were applied on $166 \mathrm{HNC}$ patients collected from Oncology Department of Xiangya Hospital. The radiotherapy protocols consisted of a total of 35 fractions equal to $68.25 \mathrm{~Gy}$, with 1.95 Gy exposure per day applied 5 days per week, during 7 weeks with $\mathrm{X}$-rays from a linear accelerator (Clinac 23EX, Varian, Lincolnshire, IL, USA) in the Department of Radiotherapy at Xiangya Hospital. Moreover, all irradiations were done in the container of the same size with the enamel samples submerged in physiological saline water. The distance between the "radiation source" and the samples would be $100 \mathrm{~cm}$.

\section{Test groups and interventions}

For each group, the open window area was treated with different protocols. All enamels were submerged in physiological saline solution and the normal saline was changed every day. The researcher who conducted the interventions of 8 experimental groups was not participated in the statistical analysis.

Group 1 (Control group): The enamels were not treated with irradiation or any anti-caries agents.

Group 2 (Irradiation): The enamels were irradiated but not treated with any anti-caries agents.

Group 3 (Irradiation+Fluoride): Every-time before irradiation, fluoride varnish (Clinpro $^{\mathrm{TM}}$ White Varnish, 3M, Saint Paul, MN, USA) was applied on enamels and left for $3 \mathrm{~min}$ then cleaned with normal saline. When the maximum cumulative radiation dose of 68.25 Gy was delivered, fluoride varnish was continued applied 5 days per week for 4 weeks.

Group 4 (Irradiation+CPP-ACP): Every-time before irradiation, CPP-ACP (GC Tooth Mousse ${ }^{\mathrm{TM}}$, Tokyo, Japan) was applied on enamels and left for 3 min then cleaned with normal saline. When the maximum cumulative radiation dose of 68.25 Gy was delivered, fluoride varnish was continued applied 5 days per week for 4 weeks.

Group 5 (Irradiation+Fluoride+CPP-ACP): Everytime before irradiation, fluoride varnish was applied on enamels and 5 min later CPP-ACP was used. When the maximum cumulative radiation dose of 68.25 Gy was delivered, fluoride varnish and CPP-ACP was continued applied 5 days per week for 4 weeks.

Group 6 (Irradiation+Infiltration resin): When the maximum cumulative radiation dose of 68.25 Gy is reached, in accordance with the manufacturer's instructions ${ }^{20)}$, the enamels were etched with 15\% HCL gel for $120 \mathrm{~s}$, rinsed for $30 \mathrm{~s}$, and dehydrated with $100 \%$ ethanol for $30 \mathrm{~s}$. Infiltration resin (Icon ${ }^{\mathrm{TM}}$, DMG, Hamburg, Germany) was applied with a micro brush for $3 \mathrm{~min}$, excess material was gently removed by air blowing and flossing, then the resin was light-cured for $40 \mathrm{~s}$. Infiltration resin was applied a second time for an additional 1 
min, light curing for $40 \mathrm{~s}$.

Group 7 (Irradiation+Infiltration resin+Fluoride): When the maximum cumulative radiation dose of 68.25 Gy was delivered, infiltration resin was applied accordance to the manufacturer's instructions then fluoride varnish was used 5 days per week for 4 weeks.

Group 8 (Irradiation+Infiltration resin+CPP-ACP): When the maximum cumulative radiation dose of 68.25 Gy was delivered, infiltration resin was applied accordance to the manufacturer's instructions then CPP-ACP was used 5 days per week for 4 weeks.

\section{SEM analysis ${ }^{21)}$}

Five enamels from each group were fixed in $2 \%$ glutaraldehyde in cacodylate buffer for $2 \mathrm{~h}$ and were dehydrated in a series of increasing ethanol concentrations (30, 50, 75, 95, and 100\%) for $10 \mathrm{~min}$. Subsequently, specimens were sputter-coated with gold in a vacuum metallizing machine (SCD500, BAL-TEC, Leica, Switzerland) and examined with a scanning electron microscope (JSM-6490LV, Neptune Tex's HP, Shimadzu, Kyoto, Japan). Two images were obtained of each sample at two magnifications $(\times 2,000$ and $\times 5,000)$.

\section{Microhardness testing}

Surface hardness of 10 enamels from each group was determined using a microhardness tester with a Vickers diamond indenter ${ }^{18)}$ (HMV-2T Microhardness Tester, Shimadzu, Kyoto, Japan). The baseline surface microhardness $\left(\mathrm{SMH}_{0}\right)$ and microhardness postirradiation $\left(\mathrm{SMH}_{1}\right)$ of all enamels were measured.

\section{Statistical analysis}

All tests employed $\alpha=0.05$ as level of significance and statistical analysis was performed with IBM SPSS Statistics Version 22 for Windows. Student's $t$ test was used to compare the microhardness pre-and postirradiation within groups. One-way analysis of variance (ANOVA) was used for comparison between groups. LSD test was used for pairwise comparison between groups when ANOVA test was significant.

\section{RESULTS}

\section{SEM analysis}

Figure 1 shows the representative SEM photographs $(\times 2,000$, insets $\times 5,000)$ of enamel surface from different groups. SEM images show that in sound enamel, it is possible to note normal organization of enamel, showing a flat and smooth surface with some striation due to the grinding procedure (Fig. 1a). The well-defined structure of enamel was destroyed after irradiation exposure with 68.25 Gy, showing enamel suffered increased porosity and cracks as depicted in Fig. 1b. Moderate superficial alterations were found in enamel applied with fluoride (Fig. 1c) and CPP-ACP (Fig. 1d), which was characterized by superficial porosities and slight depressions. Following application of fluoride with CPP-ACP (Fig. 1e), the enamel surface was distributed with small globular or rod-shaped mineral particles with high-density, forming irregular cloud or snowlike mineralized structure. The enamel morphology of infiltration resin group was slightly changed (Fig. 1f). The surface was well-distributed and smooth and only a few shallow pits and cracks were observed. The micrographs for infiltration resin plus fluoride (Fig. 1g) or CPP-ACP (Fig. 1h) were similar. The enamel surfaces were slightly changed and covered by large number of
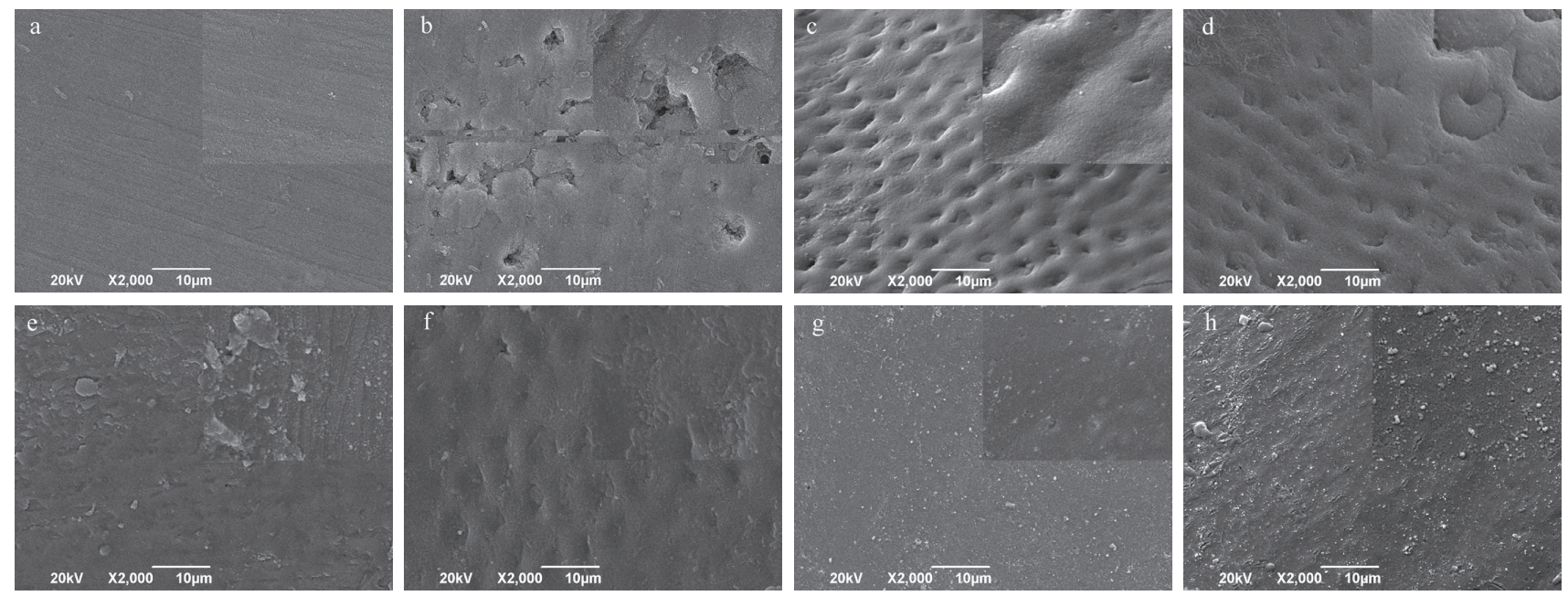

Fig. 1 Representative SEM photographs of enamel surface appearance from different groups $(\times 2,000$, insets $\times 5,000)$ : a. Control group; b. Irradiation group; c. Irradiation+Fluoride; d. Irradiation+CPP-ACP; e. Irradiation+Fluoride+CPPACP; f. Irradiation+Infiltration resin; g. Irradiation+Infiltration resin+Fluoride; h. Irradiation+Infiltration resin+CPP-ACP. 
Table 1 Microhardness value of permanent enamel treated with different anti-caries agents, and results of Student's $t$ test pre-and post-irradiation intra-groups $\left(\bar{X}_{ \pm} S, n=10, \mathrm{MHV}\right)$

\begin{tabular}{ccccc}
\hline Groups & $\mathrm{SMH}_{0}\left(\mathrm{Kg} / \mathrm{mm}^{2}\right)$ & $\mathrm{SMH}_{1}\left(\mathrm{Kg} / \mathrm{mm}^{2}\right)$ & $t$ & $p$ \\
\hline G1 & $336.80 \pm 9.61$ & $336.92 \pm 9.82$ & -0.13 & 0.905 \\
G2 & $332.53 \pm 7.55$ & $263.86 \pm 7.88$ & 28.37 & $0.00^{*}$ \\
G3 & $335.55 \pm 4.35$ & $286.70 \pm 10.97$ & 16.15 & $0.00^{*}$ \\
G4 & $336.29 \pm 10.35$ & $292.25 \pm 10.60$ & 8.26 & $0.00^{*}$ \\
G5 & $337.30 \pm 10.82$ & $315.43 \pm 10.50$ & 10.61 & $0.00^{*}$ \\
G6 & $335.79 \pm 8.47$ & $304.81 \pm 13.58$ & 5.90 & $0.00^{*}$ \\
G7 & $336.28 \pm 7.06$ & $320.42 \pm 5.77$ & 4.57 & $0.00^{*}$ \\
G8 & $338.02 \pm 9.92$ & $320.04 \pm 9.89$ & 4.75 & $0.00^{*}$ \\
\hline
\end{tabular}

*means that there was significant difference in the enamel microhardness pre- and post-irradiation intra-group ( $p<0.05)$. SMH: surface microhardness.

Table 2 Microhardness value of permanent enamel treated with different anti-caries agents, and results of one-way ANOVA analysis pre-and post-irradiation between-groups $\left(\bar{X}_{ \pm} S, n=10, \mathrm{MHV}\right)$

\begin{tabular}{|c|c|c|c|c|c|c|}
\hline Groups & $\mathrm{SMH}_{0}\left(\mathrm{Kg} / \mathrm{mm}^{2}\right)$ & $\mathrm{SMH}_{1}\left(\mathrm{Kg} / \mathrm{mm}^{2}\right)$ & $F_{1}$ & $P_{1}$ & $F_{2}$ & $P_{2}$ \\
\hline G1 & $336.80 \pm 9.61$ & $336.92 \pm 9.82$ & \multirow{8}{*}{0.23} & \multirow{8}{*}{0.98} & \multirow{8}{*}{37.04} & \multirow{8}{*}{$0.00 *$} \\
\hline G2 & $332.53 \pm 7.55$ & $263.86 \pm 7.88$ & & & & \\
\hline G3 & $335.55 \pm 4.35$ & $286.70 \pm 10.97$ & & & & \\
\hline G4 & $336.29 \pm 10.35$ & $292.25 \pm 10.60$ & & & & \\
\hline G5 & $337.30 \pm 10.82$ & $315.43 \pm 10.50$ & & & & \\
\hline G6 & $335.79 \pm 8.47$ & $304.81 \pm 13.58$ & & & & \\
\hline G7 & $336.28 \pm 7.06$ & $320.42 \pm 5.77$ & & & & \\
\hline G8 & $338.02 \pm 9.92$ & $320.04 \pm 9.89$ & & & & \\
\hline
\end{tabular}

$F_{1}, P_{1}$ : One-way ANOVA analysis in enamel SMH between 8 groups before irradiation. $F_{2}, P_{2}$ : One-way ANOVA analysis in enamel SMH between 8 groups after irradiation. SMH: surface microhardness. *means that there was significant difference in enamel SMH post-irradiation between 8 groups $(p<0.05)$.

Table 3 Microhardness value of permanent enamel treated with different anti-caries agents, and results of LSD- $t$ pairwise comparison test post-irradiation between-groups $\left(\bar{X}_{ \pm} S, n=10, \mathrm{MHV}\right)$

\begin{tabular}{ccccccccccc}
\hline Groups & $\mathrm{SMH}_{1}\left(\mathrm{Kg} / \mathrm{mm}^{2}\right)$ & $P_{1}$ & $P_{2}$ & $P_{3}$ & $P_{4}$ & $P_{5}$ & $P_{6}$ & $P_{7}$ & $P_{8}$ \\
\hline G1 & $336.92 \pm 9.82$ & - & 0.00 & 0.00 & 0.00 & 0.00 & 0.00 & 0.00 & 0.00 \\
G2 & $263.86 \pm 7.88$ & 0.00 & - & 0.00 & 0.00 & 0.00 & 0.00 & 0.00 & 0.00 \\
G3 & $286.70 \pm 10.97$ & 0.00 & 0.00 & - & 0.31 & 0.00 & 0.00 & 0.00 & 0.00 \\
G4 & $292.25 \pm 10.60$ & 0.00 & 0.00 & 0.31 & - & 0.02 & 0.00 & 0.00 & 0.00 \\
G5 & $315.43 \pm 10.50$ & 0.00 & 0.00 & 0.00 & 0.02 & - & 0.06 & 0.01 & 0.01 \\
G6 & $304.81 \pm 13.58$ & 0.00 & 0.00 & 0.00 & 0.00 & 0.06 & - & 0.36 & 0.40 \\
G7 & $320.42 \pm 5.77$ & 0.00 & 0.00 & 0.00 & 0.00 & 0.01 & 0.36 & - & 0.94 \\
G8 & $320.04 \pm 9.89$ & 0.00 & 0.00 & 0.00 & 0.00 & 0.01 & 0.40 & 0.94 & - \\
\hline
\end{tabular}

$P_{\mathrm{n}}$ means that results of LSD- $t$ test between Gn and other 7 groups $(n=1-8)$. SMH: surface microhardness. *means that there was significant difference in enamel $\mathrm{SMH}$ post-irradiation between-groups $(p<0.05)$. 
irregular high-density minerals.

\section{SMH analysis}

The SMH values of enamel for all groups pre-and postirradiation were presented in Table 1 . The results of Student's $t$ test pre-and post-irradiation intra-groups showed that irradiation significantly reduced the $\mathrm{SMH}$ of enamel $(p<0.05)$. One-way ANOVA analysis showed that no significant difference was found in enamel $\mathrm{SMH}$ between 8 groups pre-irradiation (Table 2, $p>0.05$ ). However, a statistically significant difference was found in SMH post-irradiation between the groups (Table $3, p<0.05$ ). Six different anti-caries agents (three applications alone and three pairwise combined applications) significantly increased the $\mathrm{SMH}$ of irradiated enamel $(p<0.05)$. However, they cannot restore the $\mathrm{SMH}$ of irradiated enamel to non-irradiation level. The order of enamel SMH after radiotherapy from the highest to the lowest was as follows: G1 (control group) $>\mathrm{G} 8$ (infiltration resin+CPP-ACP) $\sim \mathrm{G} 7$ (infiltration resin+ fluoride) $\sim \mathrm{G} 6$ (infiltration resin) $\sim \mathrm{G} 5$ (fluoride+CPP-ACP) $>\mathrm{G} 4 \quad(\mathrm{CPP}-\mathrm{ACP}) \sim \mathrm{G} 3$ (fluoride) $>\mathrm{G} 2$ (irradiation group). The $\mathrm{SMH}$ values of G5-G8 were the highest among 7 irradiated enamel group, and LSD- $t$ test showed that no statistically significant difference was detected between them (Table 3, p>0.05). G3 and G4 groups were not statistically different as well (Table $3, p>0.05)$.

\section{DISCUSSION}

The aim of this study was to investigate the preventive effects of different anti-caries procedures on radiationcaries by simulating the influences of irradiation on the micromorphology and mechanical properties of enamel in vitro. It is indeed that the radiation affected the structural and mechanical properties of enamel. The infiltration resin, CPP-ACP and fluoride, whether used alone or in combination with each other, can effectively improve the destruction of enamel surface caused by irradiation, and increase its surface microhardness. Among which the infiltration resin and its combination with CPP-ACP or fluoride had the most remarkable effects on the prevention of radiation-caries. The null hypothesis was rejected.

Radiation-caries is the most common oral complication after radiotherapy in HNC patients. SEM is widely used to elucidate microstructural changes in the surface of dental hard tissues after being treated with different anti-caries procedures ${ }^{22)}$. Microhardness is one of the important physical properties of tooth surface, which reflects the ability to resist plastic deformation and persistent indentation. Featherstone ${ }^{23)}$ found that a linear correlation was between microhardness and enamel mineral content. Therefore, the determination of enamel microhardness and SEM has become important measures and indexes to estimate the demineralization and remineralization of dental hard tissues in the studies of caries researches.

The Vickers microhardness of enamel is generally in the range of $254 \sim 363\left(\mathrm{~kg} / \mathrm{mm}^{2}\right)^{24)}$. In this study, the baseline SMH of enamel was $321.34-353.68 \mathrm{~kg} / \mathrm{mm}^{2}$, which was in accordance with the range of normal enamel microhardness. After irradiation, decreases in microhardness values accompanied with superficial morphology alterations were found, which was consistent with previous studies ${ }^{3,19)}$. Radiotherapy has significantly destructive effects on the organic matrix in enamel, which influences the mechanical properties of enamel ${ }^{19)}$. Liang ${ }^{25)}$ showed that the mechanism of weakened microhardness caused by irradiation was the decarboxylic reaction of dental hard tissues. Radiation induces carboxylate decarboxylation on the side chain of collagen, resulting in the loss of acidic phosphates. In addition, decrease of apatite crystals-organic matrix interaction and increase of carbon dioxide production create microcracks and fractures in hydroxyapatite minerals, resulting in rough enamel surface and decreased microhardness ${ }^{26)}$. Fränzel and Gerlach ${ }^{4}$ reported that the deleterious effects of irradiated dental hard tissues seemed like the process of demineralization. These changes of micromorphology leaded to the reduced microhardness values.

If these lesions are properly treated, enamel caries can be arrested, hardened and reverted to healthy enamel condition through a remineralization process involving the diffusion of minerals into defective tooth structures. Early detection and remineralization of demineralized dental tissues through application of oral hygiene instruction are important methods to prevent radiationcaries in HNC patients. We would like to encourage different protocols to minimize damages caused by radiotherapy on enamel and reduce this impact during and after radiotherapy. Three agents were applied solely or combined with each other on irradiated enamel and we have noted that each treatment was to some extent effective. Infiltration resin is a new method for limiting demineralization of dental hard tissues. After light curing, the resin-porous hydroxyapatite complex was formed, which occludes micro-porosities within the caries lesion, forming a diffusion barrier to prevent further demineralization ${ }^{27}$. After the application of infiltration resin, the mineral content of the demineralized enamel was only $2.1 \%$ less than that of sound enamel ${ }^{28)}$. In this study, infiltration resin was used for the first time in preventing radiation-caries and achieved significant curative effects. When the tested enamel was treated with one of infiltration resin, CPP-ACP or fluoride, it was found that infiltration resin had the strongest effects on the maintenance of micromorphology and the increasement of microhardness of irradiated enamel. In agreement with our findings, previous studies reported that the effects of infiltration resin on the surface microhardness of demineralized enamel was better than that of fluoride ${ }^{29)}$ and $\mathrm{CPP}-\mathrm{ACP}^{30)}$, which was closely related to the highly permeability of infiltration resin.

It is well known that the remineralized layer formed by fluoride or CPP-ACP can seal the enamel surface, but only a small amount can enter into the demineralized micropores of enamel with a very shallow permeation depth ${ }^{31)}$. However, infiltration resin can 
penetrate deep into the inner space of demineralized enamel. Its average penetration depth is $516.8 \mu \mathrm{m}$, and the deepest penetration depth is $973 \mu \mathrm{m}^{27)}$, which forms a diffusion barrier and blocks the passage of acid into enamel. Then the structure of demineralized enamel was significantly improved and the mechanical properties strengthened. Souza ${ }^{32)}$ determined the surface microhardness of infiltration resin samples and found that its microhardness was not only lower than that of sound enamel, but also lower than that of demineralized enamel. Therefore, the effectiveness of infiltration resin on irradiated enamel is not because of the increase in microhardness of infiltration resin material after curing itself, but its penetration into the micropores within the enamel crystals and formation of a mixture layer of enamel-infiltration resin. The restoration of enamel superficial morphology and strengthening of mechanical supports resulting in the rise of microhardness.

In this study, CPP-ACP and fluoride had protective effects on irradiated enamel. Fluoride ions combine with hydroxyapatite to produce fluorapatite, a strong deterrent to demineralization, which deposits on the surface or micropores of enamel. The crystal structure of fluorapatite is more stable than that of pure hydroxyapatite, which increases the ability of fluoride to resist incipient caries $^{33)}$. CPP-ACP has been shown to localize and stabilize calcium and phosphate ions at the enamel surface in bioavailable forms that can promote remineralization of enamel lesions ${ }^{8}$. Zhou ${ }^{34)}$ have demonstrated that by increasing the grain size of hydroxyapatite and the mol ratio of calcium to phosphorus, CPP-ACP effectively repaired the microstructure of enamel. In our study, no statistically significant differences were found between the commercially available CPP-ACP and fluoride on microhardness of irradiated enamel. Hay and Thomson ${ }^{35)}$ compared the anti-caries effects of CPP-ACP and fluoride for HNC patients after radiotherapy and believed that both CPP-ACP and fluoride can effectively prevent radiation-caries, but no significant differences were found between them. In addition, because CPPACP was as effective as fluoride, the application of CPPACP may have advantages in HNC patients due to the fact that it can help reduce the risk of radiation-caries without increasing the risk of fluorosis.

In this study, the protective effects of infiltration resin combined with fluoride or with CPP-ACP on irradiated enamel was the most obvious among 8 groups. Previous studies ${ }^{16,36)}$ have combined infiltration resin with fluorides to treat early caries and found their synergetic anti-caries effects. And the combination of infiltration resin with CPP-ACP was better than that of CPP-ACP in reducing demineralization and facilitating remineralization of dental hard tissue ${ }^{17)}$. In this study, the protective effects of infiltration resin combined with fluoride or with CPP-ACP on irradiated enamel were stronger than infiltration resin monotherapy, but no significant difference were found between them. This may be due to the permanent occlusion of enamel micropores and surface by infiltration resin, which makes fluoride and CPP-ACP unable to interact with hydroxyapatite. As a result, fluoride or CPP-ACP are mainly deposited on the surface of light-cured infiltration resin. Therefore, when infiltration resin combined with fluoride or with CPP-ACP, the infiltration resin plays a leading role in protecting irradiated enamel.

Studies ${ }^{11,37)}$ demonstrating that adjunct use of CPPACP with stannous fluorides achieved significantly clinical advantages over CPP-ACP or fluorides alone in reducing the progression of radiation-caries in patients with NPC, which was consistent with our study. In a randomized clinical trial ${ }^{11}$ of 24 NPC patients, the patients treated with both CPP-ACP and fluorides were underwent lower progressive caries behavior after a follow-up period of 3 months, which confirmed that the simultaneous application of CPP-ACP with fluorides has synergistic effects in preventing radiation-caries. In the presence of fluoride, CPP-ACP mixed with fluoride ions to form a new complex: casein phosphopeptideamorphous calcium fluoride phosphate (CPP-ACFP). Patil $^{38)}$ believed that CPP-ACFP nanocomposites may produce more effective remineralization components than fluorides or CPP-ACP. In this study, fluoride was firstly applied, making the concentration of fluoride ion increased, which provides a stable substrate for CPPACP. Therefore, the current study argues that CPP-ACP should be used clinically in collaboration with fluorides rather than applied as an alternative to fluorides.

The appearance of dental fluorosis and osteofluorosis in the process of application also limited the clinical use of fluorides to some extent. No allergies or severe side effects were recorded when evaluating the clinical safety of CPP-ACP ${ }^{39}$. CPP-ACP were derived from milk protein casein, with the results that people with milk allergies are not allowed to use CPP-ACP. No side effects associated with infiltration resin have been observed so far ${ }^{40)}$. Infiltration resin and CPP-ACP have great biosafety and can be effectively used for irradiated enamel, so both of them could be widely applied in clinical as preventive procedures for radiation-caries.

This study was an in vitro experiment mainly based on permanent teeth. The conditions in vitro are crucially different from those in vivo with dynamic biological system. Therefore, this study cannot completely simulate the actual oral cavity of HNC patients after radiotherapy. As to the mechanical properties of dental hard tissues, Vickers microhardness was selected. However, other parameters such as fracture resistance, ultimate tensile strength and elastic modulus, needed to be further studied. Therefore, more in vitro, in vivo studies and clinical trials are needed to further demonstrate the remineralized potential and longterm anti-caries efficacy of infiltration resin, CPP-ACP, fluoride and their combined application of each other for the radiation-caries.

\section{CONCLUSIONS}

Based on the data from this in vitro study, we make the following conclusions: (1) The exposure of 68.95 Gy 
radiotherapy was able to affects micromorphological structure changes of enamel accompanied with reduced microhardness. (2) Infiltration resin, CPP-ACP and fluorides can effectively promote the remineralization of irradiated enamel and preserve the integrity of enamel morphology. Among them, infiltration resin has the strongest ability to prevent radiation-caries. (3) Fluoride and CPP-ACP have synergistic effect on remineralization of radiation-caries. The synergistic effects of infiltration resin combined with fluoride or CPP-ACP on radiationcaries were stronger than combined effects of fluoride and CPP-ACP, but the same with that of infiltration resin monotherapy.

\section{ACKNOWLEDGMENTS}

The authors would like to express their gratitude to DMG company, Germany for supporting Icon infiltration-resin for this research.

This work was supported by the Science and Technology Department of Hunan Province under Grant [20191176], which were not involved in study design, in the collection, analysis and interpretation of data, in the writing of the report, and in the decision to submit the article for publication.

\section{CONFLICT OF INTEREST}

The authors report no conflict of interest.

\section{REFERENCES}

1) Dobroś K, Hajtobryk J, Wróblewska M, Zarzecka J. Radiationinduced caries as the late effect of radiation therapy in the head and neck region. Contemp Oncol 2016; 20: 287-290.

2) Silva AR, Alves FA, Antunes A, Goes MF, Lopes MA. Patterns of demineralization and dentin reactions in radiation-related caries. Caries Res 2009; 43: 43-49.

3) Lieshout HF, Bots CP. The effect of radiotherapy on dental hard tissue: A systematic review. Clin Oral Investig 2014; 18: 17-24.

4) Fränzel W, Gerlach R. The irradiation action on human dental tissue by X-rays and electrons - a nanoindenter study. Z Med Phys 2009; 19: 5-10.

5) Kumar D, Rastogi N. Oral complications and its management during radiotherapy. Indian J Dent Sci 2011; 2: 109-113.

6) Soares CJ, Neiva NA, Soares PB, Dechichi P, Novais VR, Naves LZ, et al. Effects of chlorhexidine and fluoride on irradiated enamel and dentin. J Dent Res 2011; 90: 659-664.

7) Memarpour M, Fakhraei E, Dadaein S, Vossoughi M. Efficacy of fluoride varnish and casein phosphopeptide-amorphous calcium phosphate for remineralization of primary teeth: A randomized clinical trial. Med Princ Pract 2015; 24: 231-237.

8) Somasundaram P, Vimala N, Mandke LG. Protective potential of casein phosphopeptide amorphous calcium phosphate containing paste on enamel surfaces. J Conserv Dent 2013; 16: $152-156$.

9) Reynolds EC, Cain CJ, Webber FL, Black CL, Riley PF, Johnson IH, et al. Anticariogenicity of calcium phosphate complexes of tryptic casein phosphopeptides in the rat. J Dent Res 1995; 74: 1272-1279.

10) Giulio AB, Matteo Z, Serena IP, Silvia M, Luigi C. In vitro evaluation of casein phosphopeptide-amorphous calcium phosphate (CPP-ACP) effect on stripped enamel surfaces. A
SEM investigation. J Dent 2009; 37: 228-232.

11) Sim CP, Wee J, Xu Y, Cheung YB, Soong YL, Manton DJ. Anti-caries effect of CPP-ACP in irradiated nasopharyngeal carcinoma patients. Clin Oral Investig 2015; 19: 1005-1011.

12) Sleibi A, Tappuni AR, Davis GR, Anderson P, Baysan A. Comparison of efficacy of dental varnish containing fluoride either with CPP-ACP or bioglass on root caries: Ex vivo study. J Dent 2018; 73: 91-96.

13) Vanichvatana S, Auychai P. Efficacy of two calcium phosphate pastes on the remineralization of artificial caries: a randomized controlled double-blind in situ study. Int J Oral Sci 2013; 5: 224-228

14) Paris S, Schwendicke F, Keltsch J, Dörfer C, Meyer-Lueckel $\mathrm{H}$. Masking of white spot lesions by resin infiltration in vitro. J Dent 2013; 41(Suppl5): e28-34.

15) JunWei $X$. Treatment of early rampant caries with resin infiltration: a case report. Journal of Medical Frontier 2014; 22: 183-184. (in Chinese)

16) Ozgul BM, Orhan K, Oz FT. Micro-computed tomographic analysis of progression of artificial enamel lesions in primary and permanent teeth after resin infiltration. J Oral Sci 2015; 57: $177-183$

17) Chen S, QingLing Y, BaoHua L. Effect of resin infiltration combined with CPP-ACP on adolescent dental erosion in early demineralization. J Clin Stom 2017; 04: 208-211. (in Chinese)

18) Abdalla R, Niazy MA, Jamil WE, Hazzaa HA, Elbatouti AA. The role of fluoride and chlorhexidine in preserving hardness and mineralization of enamel and cementum after gamma irradiation. Radiat Environ Biophys 2017; 56: 187-192.

19) de Barros da Cunha SR, Fonseca FP, Ramos PAMM, Haddad CMK, Fregnani ER, Aranha ACC. Effects of different radiation doses on the microhardness, superficial morphology, and mineral components of human enamel. Arch Oral Biol 2017; 80: 130-135.

20) Ozgul BM1, Orhan K, Oz FT. Micro-computed tomographic analysis of progression of artificial enamel lesions in primary and permanent teeth after resin infiltration. J Oral Sci 2015; 57: 177-183.

21) Rodrigues RB, Soares CJ, Junior PCS, Lara VC, AranaChavez VE, Novais VR. Influence of radiotherapy on the dentin properties and bond strength. Clin Oral Investig 2018; 22: 875-883.

22) Elkassas D, Arafa A. Remineralizing efficacy of different calcium-phosphate and fluoride based delivery vehicles on artificial caries like enamel lesions. J Dent 2014; 42: 466474.

23) Featherstone JD, Ten Cate JM, Shariati M, Arends J. Comparison of artificial caries-like lesions by quantitative microradiography and microhardness profiles. Caries Res 1983; 17: 385-391.

24) Lata S, Varghese NO, Varughese JM. Remineralization potential of fluoride and amorphous calcium phosphate-casein phospho peptide on enamel lesions: An in vitro comparative evaluation. J Conserv Dent 2010; 13: 42-46.

25) Liang X, Zhang JY, Cheng IK, Li JY. Effect of high energy $\mathrm{X}$-ray irradiation on the nano-mechanical properties of human enamel and dentine. Braz Oral Res 2016; 30: 1.

26) Hübner W, Blume A, Pushnjakova R, Dekhtyar Y, Hein HJ. The influence of X-ray radiation on the mineral/organic matrix interaction of bone tissue: an FT-IR microscopic investigation. Int J Artif Organs 2005; 28: 66-73.

27) Prajapati D, Nayak R, Pai D, Upadhya N, V KB, Kamath P. Effect of resin infiltration on artificial caries: An in vitro evaluation of resin penetration and microhardness. Int J Clin Pediatr Dent 2017; 10: 250-256.

28) Kielbassa AM, Muller J, Gernhardt CR. Closing the gap between oral hygiene and minimally invasive dentistry: a review on the resin infiltration technique of incipient 
(proximal) enamel lesions. Quintessence Int 2009; 40: 663681.

29) Peng Y, Qian Z, Ting Z, Jie F, Xiaomei X, Li M. The effect of resin infiltration vs. fluoride varnish in enhancing enamel surface conditions after interproximal reduction. Dent Mater J 2016; 35: 756-761.

30) Dilber E, Akın M, Yavuz T, Erdem A. Effects of different demineralization-inhibiting methods on the shear bond strength of glass-ceramics. J Prosthodont 2015; 24: 407-413.

31) Bergstrand F, Twetman S. A Review on prevention and treatment of post-orthodontic white spot lesions -Evidencebased methods and emerging technologies. Open Dent J 2011; 5: $158-162$

32) Souza ROA, Özcan M, Michida SM, de Melo RM, Pavanelli CA, Bottino MA, et al. Conversion degree of indirect resin composites and effect of thermocycling on their physical properties. J Prosthod 2010; 19: 218-225.

33) Rosin-Grget K, Peros K, Sutej I, Basic K. The cariostatic mechanisms of fluoride. Acta Med Acad 2013; 42: 179-188.

34) Zhou C, Zhang D, Bai Y, Li S. Casein phosphopeptideamorphous calcium phosphate remineralization of primary teeth early enamel lesions. J Dent 2014; 42: 21-29.
35) Hay KD, Thomson WM. A clinical trial of the anticaries efficacy of casein derivatives complexed with calcium phosphate in patients with salivary gland dysfunction. Oral Surg Oral Med Oral Pathol Oral Radiol Endod 2002; 93: 271-275.

36) Ammari MM, Jorge RC, Souza I, Soviero VM. Efficacy of resin infiltration of proximal caries in primary molars: 1-year follow-up of a split-mouth randomized controlled clinical trial. Clin Oral Investig 2018; 22: 1-8.

37) Papas A, Russell D, Singh M, Kent R, Triol C, Winston A. Caries clinical trial of a remineralising toothpaste in radiation patients. Gerodontology 2008; 25: 76-88.

38) Patil N, Choudhari S, Kulkarni S, Joshi SR. Comparative evaluation of remineralizing potential of three agents on artificially demineralized human enamel: An in vitro study. J Conserv Dent 2013; 16: 116-120.

39) Sitthisettapong T, Phantumvanit P, Huebner C, Derouen T. Effect of CPP-ACP paste on dental caries in primary teeth: a randomized trial. J Dent Res 2012; 91: 847-852.

40) Altarabulsi MB, Alkilzy M, Petrou MA, Splieth C. Clinical safety, quality and effect of resin infiltration for proximal caries. Eur J Paediatr Dent 2014; 15: 39-44. 